\title{
NUEVOS DATOS ACERCA DE LA SINGULARIDAD FITOGEOGRÁFICA DE LAS SIERRAS DE ALGECIRAS (CÁDIZ): SPHAGNUM PLATYPHYLLUM EN LA SIERRA DE LA PALMA
}

\author{
Margarita ACÓN REMACHA y Carlos MORLA JUARISTI
}

\begin{abstract}
RESUMEN. Nuevos datos acerca de la singularidad fitogeográfica de las Sierras de Algeciras (Cádiz): Sphagnum platyphyllum en la Sierra de la Palma. Al examinar unas muestras de briófitos recolectadas en las proximidades de Los Barrios (Cádiz), identificamos dos especies de Sphagnum de la sección Subsecunda: S.denticulatum Brid. Sull. (= S.lescurii Sull., S.auriculatum Schimp.) y S.platyphyllum (Braithw.) Warnst. El primero ya había sido mencionado en la provincia de Cádiz, no sucediendo lo mismo con el segundo que no se conocía de Andalucía. La presencia de esta especie, de distribución fundamentalmente circumpolar (Karlin, 1991), proporciona un dato florístico más a añadir al conjunto de elementos que hacen de estas serranías meridionales gaditanas una zona de particular significación geobotánica.
\end{abstract}

Palabras clave. Sphagnum, corología, Andalucía occidental.

ABSTRACT. New data about phytogeographical singularity of Sierras de Algeciras (Cádiz): Sphagnum platyphyllum in Sierra de la Palma. The study of a bryophytes samples from the neighbourhood of Los Barrios (Cádiz) reveals the existence of two species of Sphagnum of the Subsecunda section: S. denticulatum Brid. (= S.lescurii Sull., S.auriculatum Schimp.) and S.platyphyllum (Braith.) Warnst. The first species had been already mentioned in the province of Cádiz, but the second one was unknown in Andalusia. The presence of this latter species with circumpolar distribution (Karlin, 1991) is a peculiar addition to the group of elements which a geobotanical meaning to the southern mountanious area of the province of Cádiz.

Key words. Sphagnum, chorology, Western Andalusia.

\section{INTRODUCCIÓN}

El conjunto de relieves formados por las areniscas oligocenas que afloran en el sur de la provincia de Cádiz constituyen la unidad geológica del Aljibe. El interés geobotánico de este territorio es conocido de antiguo, Font Quer (1927) ya lo destacaba: «Yo no conozco en España país tan interesante por su flora desde el punto de vista geográfico, como las montañas de Algeciras hasta Alcalá de los Gazules». En los años 70 nuevos datos contribuyeron a incrementar la significación biogeográfica de este territorio (Molleswort Allen, 1971, 1976). Las referencias a la misma son habituales en los trabajos botánicos que 
sobre la zona se han venido efectuando (Ceballos, 1929; Pau, 1922; Díez Garretas et al., 1986, Guerra et al., 1980, Gil y Guerra, $1981)$ etc.

Los aspectos más destacables de dicha singularidad pueden resumirse en:

$\left.1^{\circ}\right)$ Existencia de un conjunto de táxones de afinidad tropical o subtropical que son interpretados como relictos de periodos pasados en los que el clima general de esta parte de Europa era más cálido y húmedo.

$2^{\circ}$ ) Importancia del elemento florístico eurosiberiano en un marco ambiental netamente mediterráneo. El relevante papel del aliso en la vegetación ribereña así como la la presencia de un significativo contingente de elementos subatlánticos, de distribución peninsular fundamentalmente noroccidental, constituyen una expresiva muestra de ello. Es precisamente dentro de éste contingente florístico donde se encuadran y encuentran significación fitogeográfica las aportaciones corológicas que se realizan en el presente trabajo.

\section{RESULTADOS}

Dos especies del género se encuentran presentes en el macizo: S.denticulatum Brid. y S. platyphyllum (Braithw.) Warnst.

Del primero ya se conocían localidades de varios puntos de la provincia de Cádiz: Picacho de Alcalá en Algeciras (Font-Quer, 1927), Sierra de Algeciras (Allorge y Allorge, 1945), Sierra de la Luna (Allorge, in FontQuer, 1950). Nosotros lo hemos herborizado en una fuente próxima al Collado de la Zarza, Los Barrios, a $380 \mathrm{~m}$ de altitud, acompañado de Fissidens serrulatum Brid., Plagiomnium undulatum (Hedw.) T.Kop. y Thammobryum alopecurum (Hedw.) Gang.

S. platyphyllum (Braithw.) Warnst., por el contrario, constituye la primera indicación para el macizo. El especimen fue recolectado en las faldas de la Sierra de la Palma, sobre una pared rezumante a $370 \mathrm{~m}$ de altitud. La formación vegetal dominante en los alrededores es un alcornocal con sotobosque de Quercus lusitanica Lam. Tras la revisión bibliográfica realizada, esta localidad parece ser la primera cita del taxon para la provincia de Cádiz y también para Andalucía, ampliándose considerablemente con ella su área de distribución. Asimismo representa el límite meridional de la misma en la Península Ibérica y Europa.

La primera indicación peninsular de esta especie se debe a Allorge \& Richard (1956) que la recolectaron en el Puerto de Cotos, Peñalara (30T VL 12), siendo efectuada la determinación por E.M. Lobley. En ese mismo trabajo se recogen dos citas anteriores (no publicadas) de los esposos Allorge, pertenecientes a la provincia de Lugo: Gandara Grande, Fereirra de Valle del Oro (29 TP J 22) y Fonsagrada (29 T PH 57), correspondiente a recolecciones de los años 1926 y 1933 respectivamente. Incorporando nuestra cita a estas localidades y al conjunto de las existentes en el resto de la bibliografía así como a las que hemos recogido en el herbario de la Briotheca Hispanica $(\mathrm{BH})$, las referencias completas del taxon en la Península se concretan en:

ASTURIAS: TP4, Faro de Peñas, Simó y Alonso, 1973 (in Simó y Alonso, 1974). CÁDIZ : TF4, Los Barrios, Acón, 1990 (hh,s/n). LA CORUÑA: NH3, Braña de Merelos, Curtis, Simó, 1975 (BH,619). HUESCA: YN2, El Bozuelo, Casas, 1965 (in Casas, 1986); Ibón de Bachimaña, Casas, 1965 (in Casas, 1986). LEÓN: TN3, Puerto de Pajares, subiendo al Brañillín, Simó (in Simó,1976); TN4, Peña Cuiña, Casas et al., 1984 (in Simó et al., 1987). LÉRIDA: CH2, Estany Llong, Casas, 1959 (in Casas, 1986); Estany Trascuro, Casas (in Casas, 1986); Hospital de Viella en el Valle de Arán, Casas (in Casas, 1986). CH4, Plans de Boavi, Casas (in Casas, 1986); Pont de Puntanou en la Font Roja, Casas (in Casas, 1986). LUGO: PJ2, Gándara Grande, Ferreira del Valle del Oro, Allorge \& Allorge, 1926 (in Allorge \& Richards, 1956); PH3, Fonsagrada, Allorge \& Allorge, 1933 (in Allorge \& Richards, 1956). MADRID: VL2, Puerto de Cotos, Peñalara, leg. Richards, det. Lobley, 1953 (in Allorge \& Richards, 1956).NAVARRA: WN3, Endarlaza, Bascones et al. (in Bascones, 1984). SALAMANCA: QE1, Las 
Batuecas, Las Mestas, subida al puerto del Portillo, leg. Ladero, det: Casas, 1972 (BH, 437).TERUEL: XK1, Puerto de Orihuela, leg. Casas et al., det: Simó, 1974 (in Casas et al.,1976).

\section{CONCLUSIONES}

El genero Sphagnum, de distribución fundamentalmente septentrional en Europa, está representado, ya con carácter marginal, en algunas altas montañas del sur del continente y del norte de Africa. La presencia de S.denticulatum Brid. y S.platyphyllum (Braithw.) Warnst. en la sierra de Algeciras es particularmente significativa tanto por su caracter meridional extremo en Europa, como por la baja cota altitudinal que alcanzan en estas sierras gaditanas.

Para interpretar su presencia junto a la anteriormente destacada conjunción de elementos florísticos, hay que remontarse en el tiempo probablemente hasta mediados del Neógeno. En ese periodo este territorio que ofrecía ya una configuración geoestructural semejante a la actual, debía contar con una buena representación de flora tropical -bastante común por lo demás en otros puntos de la Península- (Solé de Porta y Porta, 1984; Rivas Carballo y Valle, 1986).

El periodo árido que entre 6,5 y $5 \mathrm{MA}$ (BP) marca el final del Mioceno (Mesiniense) y que pudo llegar a provocar la desecación de la cuenca mediterránea (Hsu, 1981; Bocquet, 1980; Anguita, 1988), representó sin duda una sería adversidad para dichas floras que perdieron buena parte de sus efectivos. Pero durante dicho periodo seco las sierras que nos ocupan y en general todo el occidente peninsular en contacto con el Atlántico, sufrieron en menor grado esa adversidad climática. En su seno pudieron establecerse enclaves-refugio para algunos táxones tropicales incompatibles con las condiciones ambientales que se generalizaron en gran parte del perímetro mediterráneo. Este hecho se encuentra bien ejemplificado por la distribución del hojaranzo (Rhododendron ponticum L. subsp. baeticum (Boiss. \& Reuter) Hanz.-Mazz.) que además de localizarse en éstas sierras meridionales gaditanas, presenta dos estaciones en Portugal, una al sur (Monchique) y otra en el centro (Caramulo).

Con el fin del Mesiniense (5 MA. BP) el Mediterráneo recupera su condición marina y comienza un periodo climático más favorable (Suc, 1984, 1989; Sole de Porta y Porta, 1984). Transcurrido el Plioceno se inicia una serie de periodos glaciares e interestadios microtérmicos que van a representar una seria adversidad para los restos de las floras terciarias que habían conseguido alcanzar el finiplioceno (Hammen et al., 1971) -tan sólo algunos de los elementos más sensibles a los fríos han conseguido a favor de refugios, en éste caso térmicos, alcanzar el Holoceno y nuestros días-. Pero las citadas secuencias glaciares van a permitir, por otra parte, la llegada de muchos elementos de las floras boreales, y es precisamente en ese contexto donde hay que situar la instalación de los Sphagnum que nos ocupan en los territorios meridionales gaditanos.

AGRADECIMIENTOS. Deseamos expresar nuestro agradecimiento a la Dra. Fuertes Lasala por la confirmación específica de los esfagnos.

\section{BIBLIOGRAFÍA}

ALLORGE, V. \& P. ALLORGE -1945- La végétation et les groupements muscinaux des montagnes d'Algécires. Mém. Mus. Nat. Hist. Nat., 21: 85-116. Paris.

ALLORGE, V. \& P.W. RICHARDS -1956Bryophytes collected in Spain during the Tenth I,P.E. in 1953. Veröff Geobot. Inst. Rübel, 31: 250-267. Zurich.

ANGUITA, F. -1988- Origen e Historia de la Tierra, 525 pp. Ed. Rueda, Madrid.

BASCONES, J.C. et al. -1984- Esfagnos y esfagnales de Navarra (España). Anales de Bio- 
logía, 2 (Sección especial): 201-208.

BOCQUET, C. -1980-Crise de salinité messinienne et floristique méditerranéenne. Naturalia Monspeliensia, $\mathrm{n}^{\circ}$ hors de serie:21-31.

CASAS, C. -1986 - Catálogo de los briófitos de la vertiente española del Pirineo Central y de Andorra. Collect. Bot., 16(2): 255-321.

CASAS, C. -1991-New checklist of spanish mosses. Orsis, 6:3-26.

CASAS DE PUIG, C. et al. -1976- Aportaciones al conocimiento de la flora briológica española. Notula II: La Sierra de Albarracín. Acta Phytotax. Barcinon., 21:19-41.

CASAS, C., E. FUERTES, y J. VARO -1987Aportaciones al conocimiento de la flora briológica española. Notula VII: El Valle de Cuiña (Sierra de Ancares). Act. VI Simp. Nac. Bot. Cript., 473-483.

CEBALLOS, L. -1929- Descripción de una notable asociación de plantas rupícolas. Mem. $R$. Soc. Esp. Hist. Nat., XV: 751-762.

DÍEZ GARRETAS, B., J. CUENCA, y A. ASENSI -1986- Datos sobre la vegetación del subsector Aljíbico (provincia Gaditano-OnuboAlgarviense). Lazaroa, 9:315-332.

Dirkse, J.M. \& Isoviita, P. -1986- Sphagnum denticulatum, an older name for S. auriculatum. Jour. of Bryology, 14(2):388-389.

Font i Quer, P. -1927- Notas sobre la flora gaditana. Bol. R. Soc. Esp. Hist. Nat., 27: 39-46.

Font i Quer, P. -1950- La Vegetación. In Terán, M.: Geografia de España y Portugal, pg.: 218-222, Montaner y Simón, Barcelona.

Gil, J.A. y J. Guerra -1981- Aportaciones briosociológicas ibéricas. I. Comunidades epífitas de las Sierras de Algeciras. An. Jard. Bot. Madrid, 37 (2): 703-719.

Guerra, J., J.A. Gil y J. Varo -1980- Dos briófitos nuevos para Europa continental. Bol. Soc. Brot. Sér. 2, 54:173-179.

Hammen, T. van der, T.A. Wijmstra \& W.H. Zagwijn -1971- The floral record of the late Cenozoic of Europe in: The late Cenozoic glacial ages. Ed. New Haven and London, Yale Univ. Press.391-424.

Hsu, K. -1981- ¿Se secó el mediterráneo?. In: Deriva continental y tectónica de placas, Selec. Scientific American 2a ed.,:219-230.

Karlin, E.F. -1991- Notes on Sphagnum in Costa Rica: a major range extensión for Sphagnum plathyphyllum. The Bryologist, 94(1): 51-52.

Molesworth Allen, B. -1971- Nota sobre helechos españoles. Lagascalia, 1:83-87.

Molesworth Allen, B. -1976- Notas sobre algunas plantas de la provincia de Cádiz. Lagascalia, 6(2):239-242.

Pau, C. -1922- Nueva contribución al estudio de la flora de Granada. Mem. Mus. Cien. Nat. Barc. Ser. Bot. vol. I n.1, 32 pp.

Richards, P.W. -1932- Note on the Bryophytes of the «Waterfall Valley» near Algeciras: an outpost of the atlantic flora. Rev. Bryol. Lichénol, 5:6-9.

Rivas Carballo, R. y M.F. Valle -1986- Nuevas aportaciones a la palinología del Terciario de la Cuenca del Duero, Torremormojón (Palencia). Studia Geológica, 22:133-143.

Simó, R.M. -1976- Los esfagnos y esfagnales de la Península Ibérica. I. La provincia Atlántica (primera parte). Acta Phytotax. Barcinon., 21:71-88.

Simó, R.M. y N. Alonso -1974- Panorama briológico del Cabo de Peñas (Asturias). Bol. Inst. Est. Ast., S.C., n. 19. Oviedo.

Sole de Porta, N. y J. Porta -1977- Primeros datos palinológicos del Messiniense (=Turoliense) en Arenas del Rey (prov. de Granada). Studia Geologica, XIII:67-88.

Solé de Porta, N y J. Porta -1984- Etat actuel des connaissances palynologiques du Tertiaire de L'Espagne. Revue de Paleobiologie, vol spécial: 209-219.

Suc, J.P. -1984- Origin and evolution of the mediterranean vegetatión and climate in Europe. Nature 307, n. 5950: 429-432.

Suc, J.P. -1989- Distributión latitudinal et étagement des associations végétales an Cénozoique Superieur dans l'aire ouest-méditerrannénne. Bull. Soc. Geol. France, 8(3):541-550.

Aceptado para su publicación en Julio de 1993

Dirección de los autores. M. Acón: Departamento de Biología. Facultad de Ciencias. Universidad Autónoma de Madrid. C. Morla: Departamento de Silvopascicultura, E.T.S. de Ingenieros de Montes. Universidad Politécnica de Madrid. 